\title{
Research on the Design of Automotive Body and Headlights Control System Based on CAN/LIN Bus
}

\author{
LEI Lin ${ }^{1, a}$, LIAO Xianli ${ }^{2, b}$ \\ ${ }^{1}$ School of Electronic and Information Engineering, Chengdu University , Chengdu,610106, China \\ ${ }^{2}$ School of Electronic and Information Engineering, Chengdu University , Chengdu,610106, China \\ aemail: science168@163.com, bemail:liao530li@163.com
}

\section{Keywords: Soccer Robot; Mechanical Analysis; Optimal Design}

\begin{abstract}
With the increase of electronic control units on cars,technology of vehicle buses have got wider and wider application, this paper puts forward a kind of scheme of automotive body and headlightscontrol system based on CAN/LIN bus, scheme introduce in detail the CAN/LIN network in the application of the control module, Complete the gateway between two kinds of network design, also gives a control system hardware interface circuit and software design idea of each part. Final results show, the scheme reduce car track tie inside and decline cost in the car, at the same time, reliable realize the function of the control of the car door, window, light.
\end{abstract}

\section{Introduction}

As the car function more perfect, the electronic control device is widely used in automobiles, resulting in the car harness is extremely complex, communication quality and reliability is getting worse, if still using traditional cabling methods, there will be a low reliability of signal transmission, failure rate and the cost will increase exponentially ${ }^{[1]}$.CAN bus is an advanced, full-featured, with high reliability and cost effective remote network communication control method. It is widely used in automotive control systems ${ }^{[2]}$.LIN bus is designed for low-cost serial communication network vehicle development. Usually LIN bus as the CAN bus network ${ }^{[1]}$ extension. In low transfer rate request applications, such as vehicle body controlling, the lower-cost LIN bus control scheme works properly, because LIN hardware implementation is based on an ordinary serial communication interface, the child nodes can be simulated by normal I/O ports with the timer ${ }^{[3]}$. The design combines the CAN bus and LIN bus their own characteristics, we propose a CAN / LIN bus based vehicle body light control systems, hybrid network applications, making the structural design of the car can be more flexible.

\section{System Overview}

The system consists of three components: a master node controller, two slave node controller and several vehicle light driver. The master node controller and CAN transceiver, LIN transceiver systems constitute a gateway node. The master node controller receives signals from the combination of the CAN network switches and other nodes through CAN transceiver. The master node controller to the signal analysis and processing, and then send control commands to the slave node controller, complete the information and communication CAN bus and LIN bus via the LIN bus. Send control signals from the node controller, control the corresponding driver module. This design ensures that real-time data transmission, but also takes into account the convenience of the subsequent expansion, in addition to using the LIN bus greatly reduces the system cost. On the wiring in accordance with the doors, windows, lights, position control node designed separately to ensure that the cable connection standard, saving car space, reduce electromagnetic interference. 


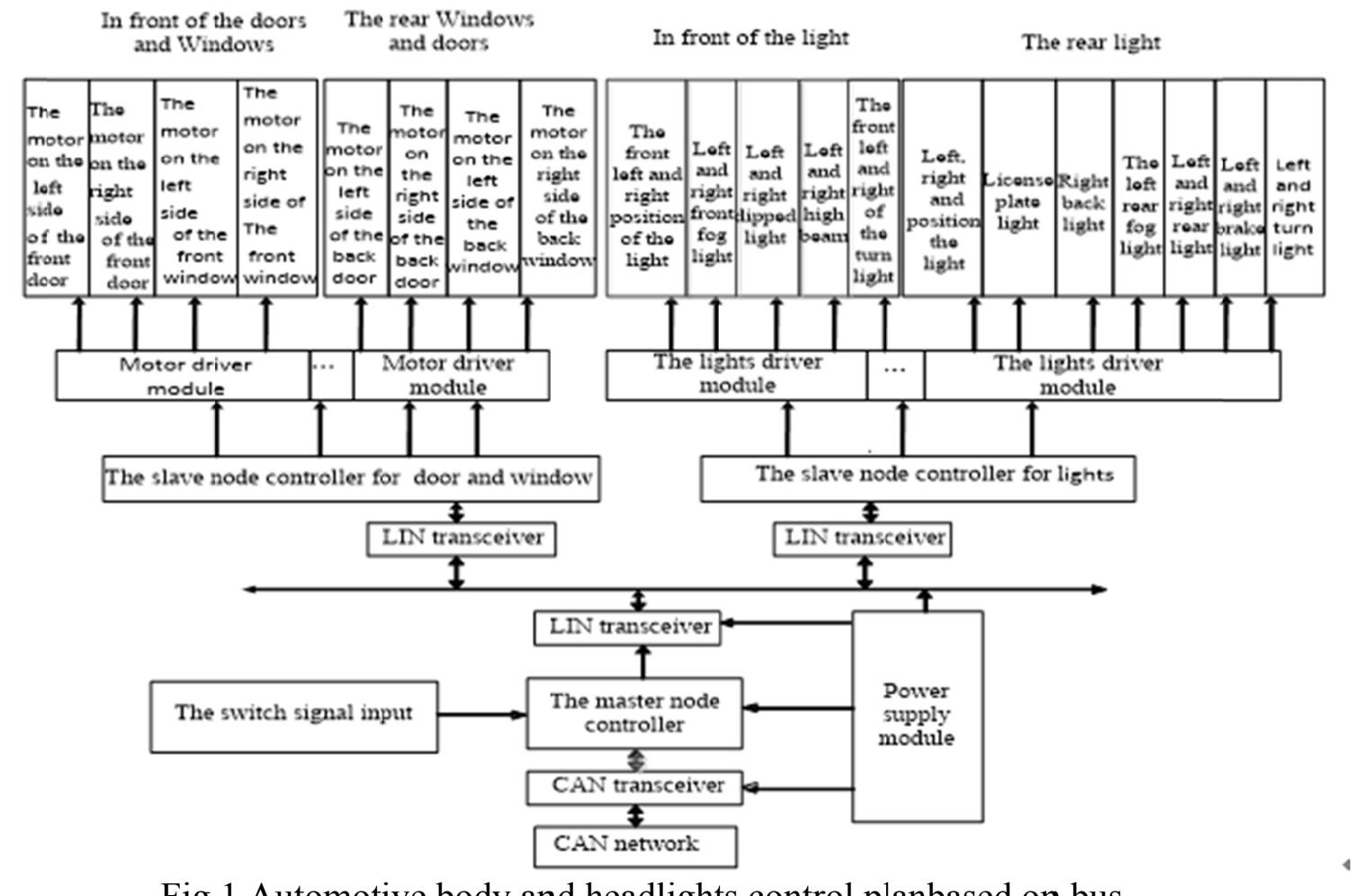

Fig.1.Automotive body and headlights control planbased on bus

\section{Hardware Design}

CAN-LIN bus master gateway node consists of three components: node controller, LIN transceiver, CAN transceiver.Master and slave node controller chip select is Freescale's 8-bit microcontrollerMC9S08DZ60. The micro-controller features an integrated multiple-functions clock generator (MCG), 24 interrupt pin, 1 dedicated input pins, 53 General purpose input/output (GPIO) pins, 2 serial communication interface (SCI) and one MSCAN communications controller ${ }^{[4]}$.

As shown in Figure 2, MC9S08DZ60 MSCAN respectively through the PTE6 and PTE7 pins and CAN-bus transceiver TJA1040 TXD and RXD pins are connected. TAJ1040 pin CANH, CANL connected CAN bus interface, this part of CAN-bus and sending and receiving data frames. MC9S08DZ60 chip in the SCI (serial communications interface is responsible for LIN communication) through the PTF0 and PTF1 pins and LIN-bus transceiver TJA1020 TXD and RXD pins are connected; TAJ1020 connected to the PIN-LIN and LIN bus interface. This part of the realization of LIN and sending and receiving data frames.

MC9S08DZ60 chip processing control unit with FIFO receive buffer module implements the storage mechanism of CAN and LIN bus data frame split, the data frame in the staging and production.

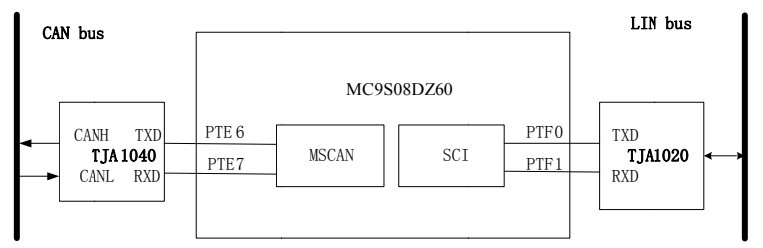

Fig.2.The gateway node hardware principle diagram

NXP's TJA1040 chip interface between the controller and the physicall bus Controller Area Network (CAN) protocol ${ }^{[5]}$.It is mainly used in high-speed applications bus speeds of up to 1Mbaud.Circuit shown in Figure 3(a), TJA1040 through TXD, RXD pin and controller chip PTE6, PTE7 pin is connected; pin CANH, CANL connected with CAN bus interface. 


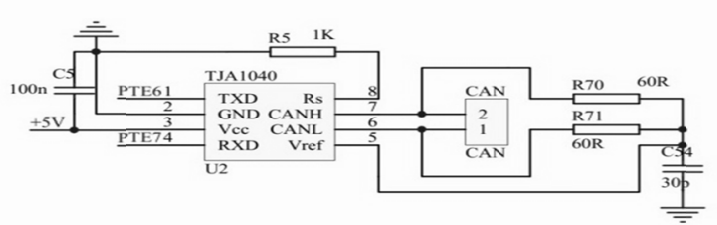

(a) CAN bus transceiver circuit principle diagram

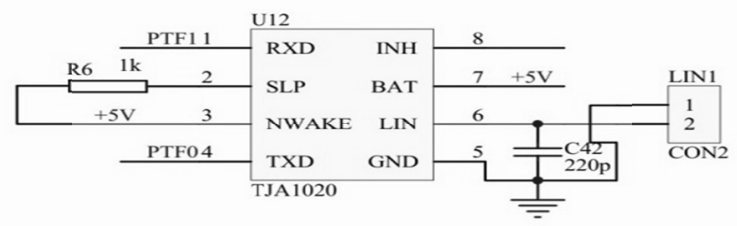

(b) LIN bus transceiver circuit diagram

Fig.3.transceiver circuit principle diagram

TJA1020 transceiver is a physical media connection, the transceiver with low power management mode ${ }^{[6]}$.Shown in Figure3(b), TJA1020 transceiver via TXD, PTF0 RXD pin and controllers, PTF1 pin connected; pin LIN and LIN bus interface is connected.LIN transceiver to connect the master and slave nodes are similar.

When selecting intelligent power driver chip and circuit design, to be selected according to various lights chip power rating. Power close to the lights we use the same chip, so the greatest degree of reduction in the number of chips, reduce circuit complexity, saves space vehicle assembly and design costs.

Due to the high beam power is $60 \mathrm{~W}$, front fog lamps power is $55 \mathrm{~W}$, near light the lamp power is $55 \mathrm{~W}^{[7]}$, these three power to close, so we choose a high-power intelligent power driver chip. Comprehensive actual vehicle lights need to be completely driven high beam, front fog lights, low beam lights need six power driver chip BTS6143D, the principle of the driving circuit portion shown in Figure 4(a).

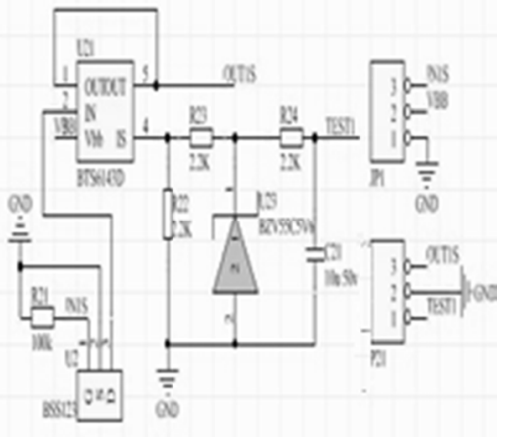

(a) Drive circuit of BTS6143D

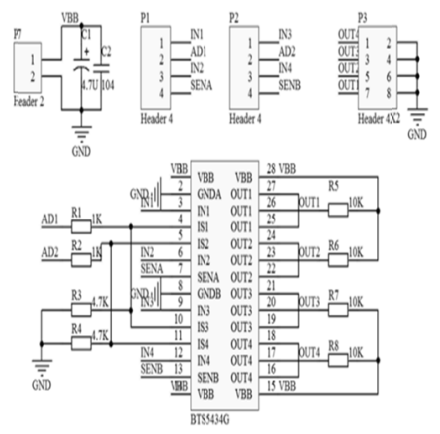

(b) Drive circuit of BTS5434G

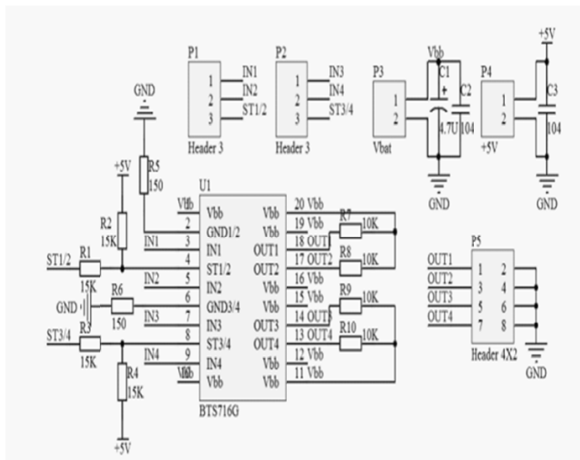

(c) Drive circuit of BTS716G

Fig.4.Schematic diagram

Although the turn lights, down lights, license plate lights and other lights of power is different, but the power is not large. The design selected Infineon series BTS5434G driver chips, a BTS5434G can take up to four loads. Comprehensive actual vehicle lights need to be completely driven turn lights, down lights, rear fog lamp, brake lights, license plate lamps require three power driver chip BTS5434G, drive circuit diagram shown in Figure 4(b).

Due to the location of lights, tail lights and side turn lamp power is $5 \mathrm{~W}$, so these three types of lights with a smart choice power driver chip. The design we chose Infineon series BTS716G driver chips, a BTS716G chip load can take up to four. Comprehensive actual vehicle lights need to be fully driven position lights, license plate lights, tail lights and side lights need two BTS716G smart power driver chip.BTS716G driving circuit schematic is shown in Figure 4(c).

Lock the motor drive circuit chose Freescale's MC33886 smart power driver chip. Shown in Figure 5(a), in order to better protect the circuit, to prevent backflow current breakdown circuit, burning the main chip, the design using opt coupler HCPL2530 protect the input circuit.HCPL2530 is a dual quick coupler, bandwidth $3 \mathrm{MHZ}$, maximum speed:1MB/S ${ }^{[8]}$. 


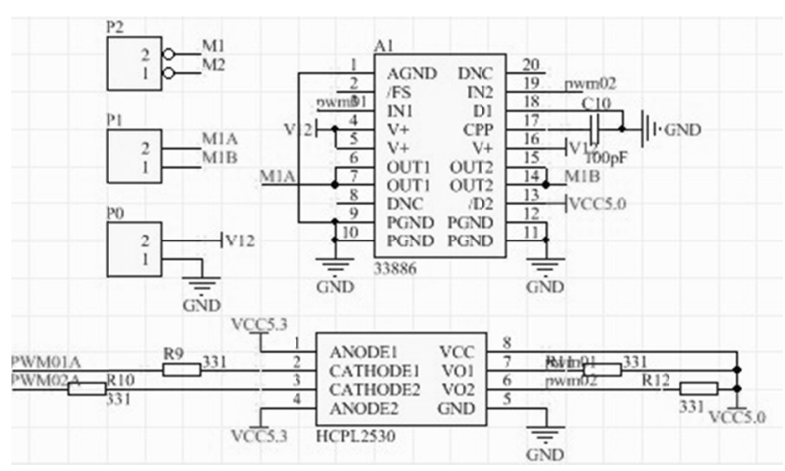

(a) MC33886 door lock motor drive

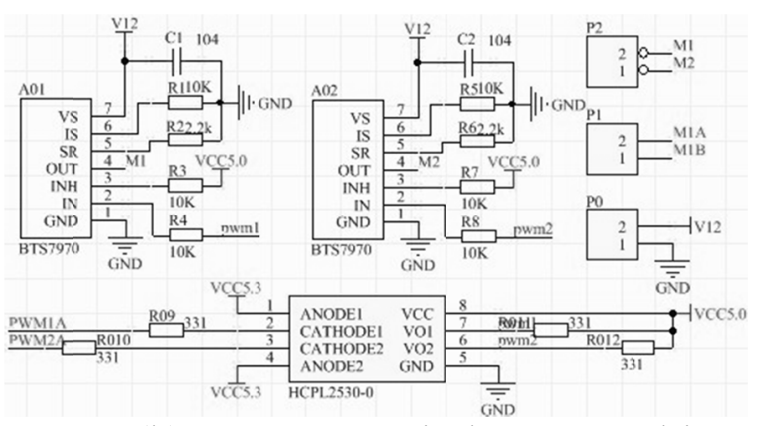

(b) BTS7960B window motor drive

Fig.5.control circuit diagram

Operation switches need to realize the four window lift control. Window motor operating current is generally about $10 \mathrm{~A}$, rise and fall time of a slight increase or decrease the current, when stall current can reach 20A.Combining various functions to achieve the windows, each window motor using independent control. The selected design intelligent power window motor driver chip is Infineon motor driver chip BTS7960, concrete drive circuit diagram shown in Figure 5(b).

\section{System software design}

Gateway node needs to implement to receive data frames from the CAN bus network, and converts the data to be transmitted into CAN LIN data frame; LIN bus network to send data as a frame to the LIN bus master node from the node; as a LIN master node receives from LIN slave nodes data frame, and converts it into CAN data frame, and forwarded to the CAN bus network; receive or send CAN data frame as a basic CAN bus network nodes to achieve a CAN node network communication functions. Shown in Figure 6, the figure on the left figure is CAN/LIN gateway program flow, the design cycle to achieve query CAN data frame is received and LIN data frame.Right side of Figure CAN data frame reception is interrupted flow chart.This design uses interrupt achieve timely reception of CAN data frame to ensure the real-time system.

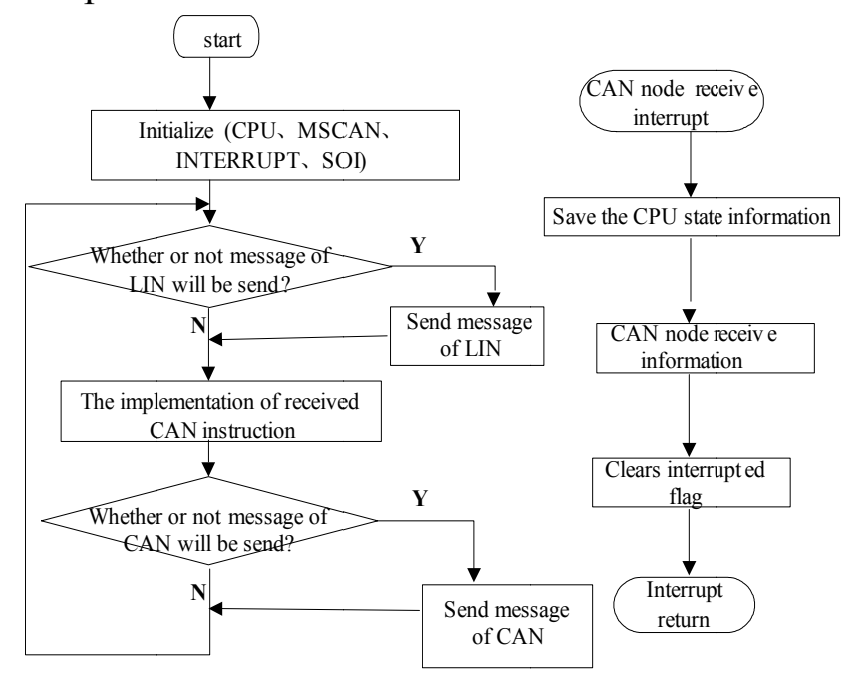

Fig.6.the CAN/LIN gateway program flow chart and interrupt flowchart

In programming CAN nodes only need to communicate via CAN transceiver CAN bus control signals into the car for the CAN bus control information.Then, LIN bus control information transmitted to the slave node, to achieve the control of the lamp and the motor.CAN node software flow chart shown in Figure 7. 


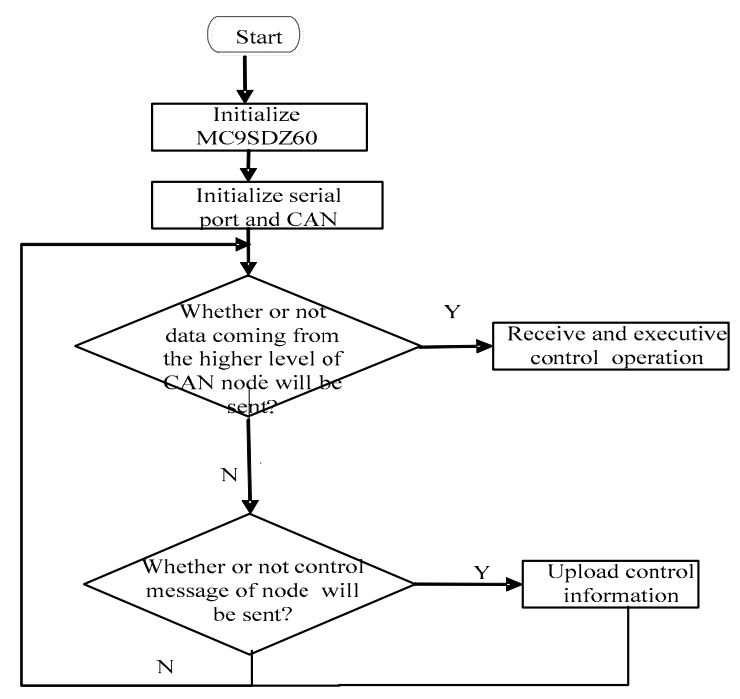

Fig.7.the CAN node software flow chart
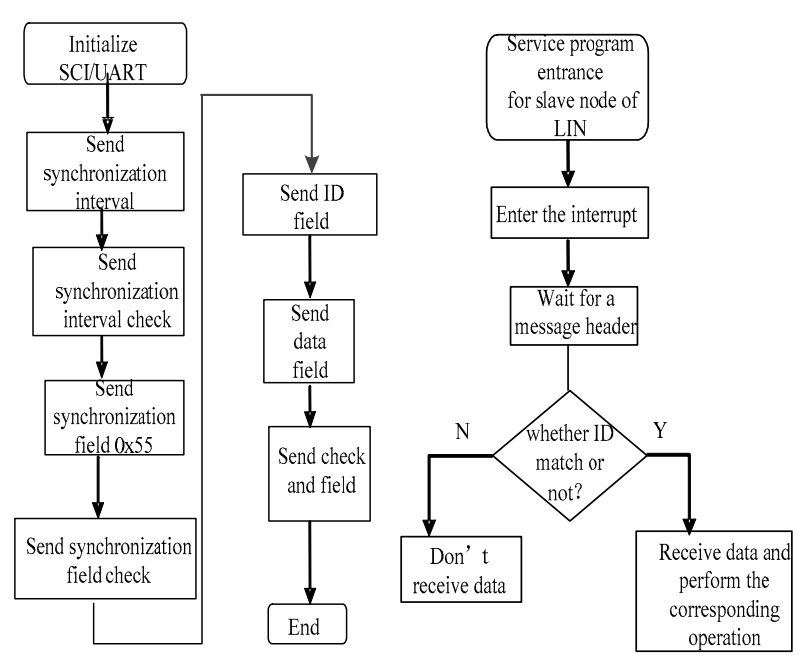

Fig.8. LIN of the main node and LIN of from the node's part of the program flow char

LIN master node core functionality of the program is to achieve a LIN bus communication.It will simulate the operation control signal is sent to the driver via the LIN slave master node.LIN slave nodes need to receive instructions from the master node, and make the appropriate action, to achieve the lights, motor controls.As shown in Figure 8, left for the LIN master node program flow, the right to LIN slave node program flow.

\section{Conclusion}

The system uses the CAN/LIN bus hybrid networks to achieve automobile headlights, door and window controls. This reduces the complexity of the vehicle network and improve the performance of the control system can effectively reduce the cost.This test system has been debugged at the carriage, the control function can be reliably completed.

\section{Acknowledgement}

In this paper, the research was sponsored by Science and Technology Support Project of Sichuan Province (Project No. 2011GZ0260)

\section{References}

[1] GuoTieqiao, Chen Yong.Intelligent Lighting System Based on CAN/LIN Bus [J]. PROCESS AUTOMATION INSTRUMENTATION，2011(02): :46-48

[2] Anon. Modeling Method of Automotive Body CAN/LIN Nets Application Protocol Based on Object-oriented Colored Petri Net[J]. Chinese Journal of Mechanical Engineering, 2011(06): 999-1006.

[3] JIANG Shu-xia, HAN Zhi-gang, ZHOU Yong-jun. In-vehicle Network Optimum Design Based on CAN/LIN Bus and CAN Communication [J].INSTRUMENT TECHNIQUE AND SENSOR, 2011 (06): :48-51.

[4] Zhu Xiaoqiang.Development of a Car's Body Control System based on CAN Bus [D]. Shanghai: Shanghai Jiao Tong University, 2010.

[5] XU Li-xian, JI Qi-chun, SU Rui. Design of electronic settlement terminal based on ARM processor and CAN bus [J]. Modern Electronics Technique, 2012 (21):167-169.

[6] LUO Xuemei,WU Qinmu. Design and Realization of Master Node and Slave Node Based on 
LIN Bus [J].MODERN ELECTRONICS TECHNIQUE，2010（05）:209-210.

[7] Li Haiyan. RESEARCH ON AUTOMOBILE BODY CONTROL SYSTEM BASED ON CAN/LIN HYBIRD NETWORK [D]. Harbin: Harbin Institute of Technology, 2012.

[8] XIA Ji-qiang, ZHANG Chuan-sen, BAI Rong-gang, et al. Real-time and reliability analysis of time-triggered CAN-bus[J]. Chinese Journal of Aeronautics, 2013(01): 171-178. 